山્યFANÇAISE

$\supset \mathrm{DE}$

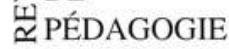

\section{Revue française de pédagogie}

Recherches en éducation

$196 \mid 2016$

Apprendre à lire et à écrire au cours préparatoire : enseignements d'une recherche collective

\title{
Les facteurs explicatifs des performances en lecture-compréhension à la fin du cours préparatoire
}

The factors which explain performance in reading comprehension at the end of the first grade of primary school

Roland Goigoux, Sylvie Cèbe et Julie Pironom

\section{(2) OpenEdition}

Journals

Édition électronique

URL : http://journals.openedition.org/rfp/5076

DOI : $10.4000 /$ rfp. 5076

ISSN : 2105-2913

Éditeur

ENS Éditions

Édition imprimée

Date de publication : 30 septembre 2016

Pagination : 67-84

ISSN : 0556-7807

Distribution électronique Cairn

\section{CAIRN INFO}

CHERCHER, REPÉRER, AVANCER.

Référence électronique

Roland Goigoux, Sylvie Cèbe et Julie Pironom, « Les facteurs explicatifs des performances en lecturecompréhension à la fin du cours préparatoire », Revue française de pédagogie [En ligne], 196 | 2016, mis en ligne le 30 septembre 2019, consulté le 30 mars 2018. URL : http://journals.openedition.org/ rfp/5076 ; DOI : 10.4000/rfp.5076 


\title{
Les facteurs explicatifs des performances en lecture- compréhension à la fin du cours préparatoire
}

\author{
Roland Goigoux \\ Sylvie Cèbe \\ Julie Pironom
}

\begin{abstract}
Des analyses de régression réalisées dans le cadre de l'enquête Lire et Écrire au CP (Ifé-ACTé) sur les performances de 2395 élèves ont révélé que les compétences en décodage et en compréhension de textes narratifs entendus contribuent les unes et les autres fortement à la compréhension d'un texte narratif lu à la fin de la première année de la scolarité élémentaire (le cours préparatoire). L'introduction de l'évaluation de compétences lexicales et phonologiques, de la fluidité de la lecture et des capacités de raisonnement non verbal permet d'affiner un modèle théorique (SVR-Texte étendu) qui peut servir d'argument pour l'action pédagogique visant l'enseignement précoce de la compréhension de textes entendus, simultanément à celui du décodage.
\end{abstract}

Mots-clés (TESE) : apprentissage, lecture, compréhension, enseignement primaire

\section{Introduction : présentation du contexte}

Au cours des trente dernières années, un consensus scientifique s'est progressivement dégagé dans le domaine de l'apprentissage initial de la lecture. On s'accorde aujourd'hui pour considérer que la compréhension de textes dépend de deux principaux ensembles de compétences : celles qui donnent accès à l'identification des mots écrits et celles qui permettent la compréhension du langage (MEN, 1992; PIREF, 2003; CNESCO, 2016). C'est pourquoi le ministère français de l'Éducation nationale demande aux maîtres d'enseigner les deux, dès le début de la scolarité obligatoire. Cette injonction, réitérée dans tous les programmes scolaires récents (1995, 2002, 2008 puis 
2015), ne s'accompagne pas toutefois d'indications précises sur les modalités pédagogiques à retenir, sans doute pour préserver la liberté pédagogique des enseignants mais aussi et surtout parce que celles-ci restent controversées tant sur le plan scientifique que professionnel. On ignore toujours, par exemple, s'il est préférable de conduire ces deux enseignements simultanément ou successivement, en interaction ou de manière indépendante.

Les résultats des recherches qui ont évalué l'influence respective de la maîtrise du code et de la compréhension du langage sur la qualité de la lecture-compréhension autonome sont souvent mobilisés comme arguments pour justifier des recommandations pédagogiques. Les synthèses francophones récentes réalisées (Sprenger-Charolles \& Colé, 2013) indiquent en effet que les habiletés de décodage sont déterminantes au début de l'apprentissage de la lecture avant que les habiletés de compréhension du langage ne deviennent «de plus en plus explicatives de la performance lorsque l'activité de lecture évolue du décodage à la compréhension des textes» (Bianco, 2010, p. 244). Ce constat ouvre la porte à deux interprétations différentes (voir les débats de la conférence de consensus organisée par le CNESCO en 2016) :

- pour les uns, défenseurs d'un modèle successif, il serait bon de commencer par focaliser tous les efforts et l'attention des élèves sur le décodage et la compréhension des mots et de différer le travail sur la compréhension des textes;

- pour les autres, défenseurs d'un modèle simultané, il serait plus pertinent de mener de front l'enseignement du déchiffrage et celui de la compréhension de textes en prenant appui, au début du cours préparatoire, sur des lectures réalisées par les enseignants.

En 2016, les documents proposés par le ministère de l'Éducation nationale pour accompagner le programme de 2015 ont tranché au profit de la seconde solution. Ils suggèrent de distinguer deux types de supports pour atteindre deux objectifs distincts (MENESR, 2016) :

- des textes que les élèves apprennent à découvrir en autonomie ("courts, aisés à décoder, simples du point de vue de la langue et des référents culturels») avec pour objectif d'articuler «les processus d'identification des mots et l'accès au sens des phrases»;

- des textes plus complexes "lus par l'adulte, comme en grande section de maternelle» pour viser le développement de compétences langagières propres à pédagogie de la compréhension aux seuls textes que les élèves peuvent déchiffrer de manière autonome.

Dans la mesure où la précision et la rapidité du décodage d'une part, et le niveau de compréhension du langage d'autre part, contribuent de façon indépendante à la compréhension en lecture (Megherbi, Seigneuric \& Ehrlich, 2006), il semble logique de travailler les deux sans attendre le cours élémentaire et sans faire dépendre l'une de l'autre. Mais personne n'a étudié quel était le meilleur dosage entre ces deux composantes de l'enseignement.

C'est dans ce contexte que s'inscrivent nos questions de recherche. Après les avoir exposées en lien avec notre cadre théorique, nous présenterons notre méthodologie puis nos résultats que nous discuterons dans notre conclusion. Ces résultats reposent sur des données que nous avons construites dans le cadre de I'enquête collective Lire et Écrire' présentée dans ce numéro de la Revue française de pédagogie, dans laquelle nous avons évalué les compétences de lecture d'un très vaste échantillon d'élèves, au début et à la fin du cours préparatoire, première année de la scolarisation élémentaire en France.

\section{Première question : la compréhension de texte dans le modèle simple de la lecture}

À la fin des années 1990, les psychologues américains Gough et Tunmer (1986) ont proposé un modèle simple de la lecture (Simple View of Reading, dorénavant SVR) dans lequel la compréhension en lecture est présentée comme le produit de deux habiletés : la reconnaissance des mots écrits et la compréhension du langage, soit $\mathrm{R}=\mathrm{D} \times \mathrm{LC}$ (Reading $=$ Decodage $\times$ Linguisitic Compréhension), chaque variable pouvant prendre une valeur comprise entre 0 et 1 (perfection). Gough et Tunmer définissent la compréhension du langage comme «le processus par lequel une information lexicale donnée, un mot, des phrases ou des discours sont interprétés » (1986, p. 7). Selon Adlof, Perfetti et Catts (2011) qui reprennent ce modèle, la compréhension est similaire "que le langage soit traité avec les oreilles ou avec les yeux». Promu en France par l'Observatoire national de la lecture (ONL, 1998), ce modèle a été traduit par la

1 La synthèse de cette enquête est en ligne en français et en anglais : <http://ife.ens-lyon.fr/ife/recherche/lire-ecrire/rapport/ synthese-du-rapport-lire-et-ecrire> (consulté le 2 février 2018). 
formule $L=D \times C L$ mais chacun des trois éléments de l'équation a donné lieu à des définitions variables selon les chercheurs :

- L représente le but de la Lecture, c'est-à-dire la compréhension de l'écrit, mais, dans un grand nombre de recherches, celle-ci est réduite à la compréhension de textes sommaires, de bribes de textes, voire de phrases isolées. Rares sont celles qui incluent, au début de l'apprentissage, à la fin du cours préparatoire par exemple, des textes impliquant une compréhension de l'implicite et donc la capacité à produire des inférences. Nous l'avons fait dans notre étude;

- D caractérise le degré de maîtrise des procédures de Décodage évalué tantôt par la lecture de pseudo-mots, tantôt par celle de mots écrits isolés, plus ou moins familiers et/ou réguliers sur le plan graphophonémique. Vitesse et précision du décodage sont cependant toujours évaluées. Nous l'avons fait aussi en combinant ces deux mesures et en en ajoutant une troisième, la lecture à haute voix de mots en contexte ;

- la définition du troisième terme, $\mathrm{CL}$, est encore plus ambiguë car la Compréhension du Langage porte, selon les auteurs, sur deux objets très différents : tantôt le langage oral (interlocution en situation de face à face : language comprehension pour Oakhill, Cain \& Elbro, 2014), tantôt le langage écrit oralisé par un tiers, c'est-à-dire au terme d'une lecture à haute voix qui dispense l'enfant de décoder les mots (listening comprehension pour Gentaz, Sprenger-Charolles $\&$ Theurel, 2015). C'est le second sens que nous retenons ici car nous n'avons pas évalué les compétences orales de réception en contexte dialogique mais celles qui sous-tendent la compréhension d'un récit écrit dont la lecture à haute voix avait été préalablement enregistrée. Dans la suite de cet article, nous appellerons donc compréhension du langage (CL) la compréhension d'un écrit (phrase ou texte) lu à haute voix par un tiers. Nous rejetterons par conséquent la traduction "compréhension orale» utilisée par d'autres chercheurs francophones car elle nous semble ambiguë : elle pourrait laisser croire qu'il s'agit de langage oral alors qu'on évalue la compréhension de langage écrit lu à haute voix.

Les modalités d'opérationnalisation du modèle SVR sont si diverses que seul l'examen des épreuves choisies pour évaluer les compétences des élèves permet de savoir à quel modèle précis se réfèrent les chercheurs. On constate, en effet, que le poids des composants du modèle varie considérablement d'une étude à l'autre en fonction des tests retenus pour quantifier
L, CL et D (Colé, Casalis, Belen Dominguez et al., 2012). On pourrait donc soutenir que la définition de la lecture dépend des tests choisis pour l'évaluer. C'est ce que concluent Keenan, Betjemann et Olson (2008) à l'issue d'une comparaison de six principaux tests d'évaluation de la compréhension montrant que la contribution respective du décodage et de la compréhension du langage varie surtout en fonction de la nature des épreuves d'évaluation de la compréhension écrite en lecture autonome (voir aussi Keenan, Olson \& Betjemann, 2009).

Toutefois, le modèle simple (SVR) permet d'expliquer pourquoi la compréhension de phrases et de textes est altérée, voire empêchée, si la maîtrise de l'un des deux composants est déficitaire et pourquoi elle est satisfaisante si les deux sont suffisants au regard du texte à lire. Lorsque le niveau du décodage est correct, le lecteur dispose des ressources cognitives nécessaires pour réaliser les traitements complexes requis par la compréhension écrite $(L)$ ce qui lui permet d'atteindre, pour celle-ci, un niveau égal à celui de la compréhension «orale » (Gersnbacher, Varner \& Faust, 1990). En d'autres termes, les déficits en compréhension orale affectent celle de l'écrit, même si les compétences de décodage sont intactes (Keenan, Betjemann \& Olson, 2008), et un décodage mal assuré nuit à la compréhension écrite même si la compréhension de I'oral est satisfaisante (Perfetti, Landi \& Oakill, 2005).

Bien que, dès 1990, Hoover et Gough aient insisté sur le fait que les deux composants du modèle SVR sont «d'égales importances» (1990, p. 128), certains chercheurs, lorsqu'ils s'adressent aux enseignants, sont tentés de privilégier le facteur qui leur semble le plus limitant dans un contexte particulier (la compréhension orale en contexte plurilingue, par exemple) ou à un moment donné de l'apprentissage (le décodage au début de l'apprentissage en langue maternelle, par exemple), le poids de ces facteurs variant sensiblement entre la première année et la sixième année d'école élémentaire (Hoover \& Gough, 1990; Ouellette \& Beers, 2010).

Notre première question de recherche porte sur les conséquences que peut avoir, sur le modèle SVR, une définition de la lecture ( $L$ ) et de la compréhension du langage $(\mathrm{CL})$ basée sur l'unité texte plutôt que sur I'unité phrase. Nous faisons l'hypothèse que, dès la fin du cours préparatoire, le poids du facteur CL est réévalué par rapport à celui de D si l'unité choisie est le texte plutôt que la phrase. En effet, pour comprendre un texte, il faut saisir le sens de chaque phrase isolée 
mais aussi construire une représentation mentale qui intègre et organise l'ensemble des propositions sémantiques en un tout cohérent. À ce sujet, les recherches de Oakhill et Cain (2012) montrent que l'utilisation du contexte est particulièrement impliquée dans le traitement de l'information textuelle et contribue à assurer la cohérence en établissant des relations sémantiques entre les phrases qui sont reliées entre elles et enchâssées dans un contexte linguistique signifiant. Ajoutons, à la suite de Florit, Roch et Levorato (2011), que la compréhension de textes lus sollicite la mobilisation de ressources cognitives attentionnelles qui ne sont pas ou sont peu requises pour comprendre des phrases isolées. Bref, la production d'inférences et I'utilisation du contexte reposent principalement sur les processus intégratifs basés sur les connaissances encyclopédiques et textuelles du lecteur qu'il est indispensable d'évaluer à la fois en $L$ et en $C L$ si l'on veut obtenir un modèle SVR fiable. Le premier modèle que nous avons testé est donc une version classique du modèle SVR, mais dans laquelle la compréhension de textes a remplacé la compréhension de phrases. Nous I'appelons modèle $\mathrm{n}^{\circ} 1$ : SVR-Texte. Ce modèle accorde une place plus importante aux compétences en compréhension du langage que les modèles SVR classiques centrés sur l'unité phrase bien que, dans les deux cas, elles restent moins déterminantes que les compétences en décodage (modèle $n^{\circ} 1$, hypothèse générale).

\section{Deuxième question : les évolutions récentes du modèle SVR}

Notre seconde question de recherche porte sur les conséquences que peut engendrer, sur le modèle SVR, une définition affinée des facteurs décodage (D) et compréhension du langage $(\mathrm{CL})$.

Si le décodage et la compréhension du langage rendent compte d'une part importante de la variance des performances en lecture, les recherches ayant validé le modèle SVR à l'aide d'analyses de régression révèlent qu'une large part de variance reste inexpliquée par ces deux seuls facteurs (Wagner, Herrera, Spencer et al., 2014). C'est pourquoi, depuis une quinzaine d'années, les chercheurs débattent sur la nécessité d'y ajouter de nouveaux composants capables de l'enrichir et d'accroître son pouvoir explicatif (Adlof, Perfetti \& Catts, 2011). Ils se demandent aussi si les deux composants ractive ou les deux à la fois. Pour répondre à cette question, la plupart s'efforcent de mieux les conceptualiser et de mieux les évaluer. Nous allons faire référence à ces débats pour justifier le second modèle que nous avons soumis à validation empirique.

Nous ne reviendrons que brièvement sur la première explication des lacunes du pouvoir explicatif du modèle SVR puisque, pour des raisons méthodologiques liées à la complexité de l'enquête Lire et Écrire, nous avons renoncé à la combler. Comme Oakhill, Cain et Elbro, nous savons que l'équation SVR «ignore le fait que les textes écrits sont, à bien des égards, très différents des interactions orales, qu'ils requièrent des compétences mémorielles et d'autres compétences cognitives qui sont moins cruciales pour comprendre les interactions de la vie quotidienne» (2014, p. 5, traduit par nous). Nous n'avons pas évalué les compétences langagières orales des enfants qui, nous l'avons dit plus haut, sont différentes des compétences langagières écrites sur plusieurs plans : elles reposent sur un traitement des données du contexte d'énonciation et des éléments non verbaux de la communication alors que l'écrit se caractérise par l'absence du référent et de I'interlocuteur. La compréhension de l'écrit exige aussi la connaissance d'un lexique plus soutenu et la maîtrise d'une syntaxe très différente de celle de l'oral. La construction de la cohésion textuelle et l'organisation des discours écrits sont donc spécifiques et les enfants doivent progressivement s'y familiariser (Bronckart, Bain, Schneuwly et al., 1985). Le traitement de l'écrit, dont la trace est permanente à la différence de l'oral éphémère, suppose également une capacité de régulation de l'activité de lecture (modulation de la vitesse, pauses, retours en arrière, détection d'incohérences, etc.) qui n'a rien à voir avec celle requise à l'oral. Pour toutes ces raisons, on ne peut prétendre inclure toutes les compétences langagières orales des enfants dans le modèle et il faut donc accepter une certaine faiblesse de son pouvoir explicatif.

Nous ne reprenons donc ici que les critiques dont nous avons su nous saisir pour enrichir le modèle initial, poursuivant en cela la voie ouverte par d'autres chercheurs qui ont proposé des modèles SVR étendus (Ouellette \& Beers, 2010 ; Colé, Casalis, Belen Dominguez et al., 2012; Tunmer \& Chapman, 2012).

\section{La fluence}

Après avoir contrôlé la précision du décodage à l'aide de tests de lecture de pseudo-mots, plusieurs cher- 
cheurs ont montré que la vitesse de décodage, évaluée par des tests de fluence, influençait la compréhension (Adlof, Catts \& Little, 2006; Tilstra, McMaster, van den Broeck et al., 2009). Aussi avons-nous introduit ce facteur dans notre second modèle et intégré un test de fluence dans notre batterie d'évaluations. Cette option pouvait paraître superflue dans la mesure où notre test initial de décodage comportait déjà un paramétrage temporel (deux épreuves de lecture en temps limité). Toutefois, il s'agissait de la lecture de mots isolés ou de pseudo-mots, ce qui ne permettait pas de rendre compte des effets exercés par le contexte dans la lecture d'énoncés. Or, plusieurs études montrent qu'il existe des écarts de performances importants entre la vitesse et la précision et que les vitesses de lecture de mots isolés et de mots en contexte sont différentes parce qu'elles ne sont pas sensibles aux mêmes paramètres (Pinnell, 1995; Adlof, Perfetti \& Catts, 2011). Bien que, dans les études disponibles, la fluence ne rende compte que d'une faible part de la variance dans la compréhension en lecture quand on contrôle le décodage et la compréhension du langage, nous avons souhaité l'évaluer car rares sont les travaux qui portent sur de très jeunes lecteurs. Si, comme Adlof et ses collaborateurs (2006) I'ont établi pour des élèves plus âgés (CE1 et CM1), la vitesse est très fortement corrélée avec la précision et modérément avec la compréhension du langage, elle n'apportera rien à nos analyses de régression. Dans le cas contraire, sa contribution sera significative parce que la lecture de mots en contexte mobilise d'autres compétences que la lecture de mots isolés. C'est ce que nous nous attendons à trouver (modèle $\mathrm{n}^{\circ} 2$, hypothèse 1 ).

\section{La phonologie}

L'importance des compétences phonologiques dans l'apprentissage de l'identification des mots écrits a été maintes fois et solidement établie (voir Sprenger-Charolles \& Colé, 2013, pour une synthèse en français). Dans la mesure où le décodage repose en grande partie sur la mise en correspondance des graphèmes et des phonèmes, on devine que la capacité à isoler et à manipuler consciemment les unités phonologiques (syllabes et surtout phonèmes) peut jouer un rôle dans la maîtrise progressive du code alphabétique et, par conséquent, dans les performances en lecture.

Reste à savoir si, dans le modèle SVR, cette contribution est indépendante du facteur décodage ou si elle y est entièrement incluse. Les spécialistes du domaine se sont posé très tôt cette question (par exemple Oaklill,
Cain et Bryant dès 2003 ou Muter et ses collaborateurs en 2004). Les résultats obtenus depuis en explorant l'extension du modèle SVR aux habiletés phonologiques apparaissent contradictoires et fluctuants selon les études, les tests retenus et l'âge des lecteurs. Nous retiendrons toutefois que c'est chez les apprentis lecteurs que l'on trouve l'influence la plus forte et la moins controversée (Vellutino, Tunmer, Jaccard et al., 2007 ; Gentaz, Spenger-Charolles, Theurel et al., 2013). C'est pourquoi nous choisissons de la tester en faisant l'hypothèse que les habiletés phonémiques sont plus déterminantes que les habiletés syllabiques (modèle $n^{\circ} 2$, hypothèse 2 ).

\section{Les habiletés cognitives non verbales}

Plusieurs études suggèrent que des compétences cognitives autres que celles qui sont requises pour traiter le langage sont impliquées dans la compréhension en lecture. L'étude de Cutting, Materek, Cole et alii (2009) montre, par exemple, que les élèves âgés de 9 à 14 ans qui présentent des déficits spécifiques en compréhension sans avoir de problème de décodage obtiennent des résultats significativement inférieurs à ceux de leurs camarades dans les tâches impliquant un fonctionnement exécutif non verbal qui requiert planification, organisation et contrôle (par exemple un labyrinthe). Compton, Fuchs, Fuchs et alii (2008) ont aussi observé l'influence du QI non verbal sur la compréhension de l'écrit, en sus des compétences langagières. Leurs résultats confortent ceux répertoriés dans la revue de questions de Fuchs et Young (2006) qui conclut au pouvoir prédicteur du QI non verbal sur la compréhension, mais pas sur le décodage. Ils sont aussi concordants avec ceux de Stuebing, Barth, Molfese et alii (2009) qui montrent que les compétences cognitives non verbales ne sont pas prédictives des effets des interventions précoces centrées sur le décodage. Autrement dit, si une faible efficience cognitive n'empêche pas d'apprendre à décoder, elle affecte la compréhension en lecture, indépendamment du niveau linguistique des enfants. C'est pourquoi nous avons ajouté une évaluation d'habiletés cognitives non verbales (via un test de raisonnement non verbal emprunté à Khomsi, 1992)² dans notre second modèle,

2 Cette épreuve visait à évaluer le niveau de « raisonnement non verbal» de l'enfant qui devait abstraire une relation entre les éléments présents dans un ensemble ordonné de trois figures géométriques pour compléter une quatrième case vierge en choisissant parmi cinq propositions. Le raisonnement portait sur des contenus figuratifs familiers qui limitaient la contribution de la 
en plus des habiletés phonologiques et de la fluence. Nous faisons I'hypothèse que ces habiletés ont un poids statistiquement significatif dès lors que nous évaluons des compétences à produire des inférences pour comprendre des récits comportant des informations implicites (modèle $n^{\circ} 2$, hypothèse 3 ).

\section{Les connaissances du lecteur et le vocabulaire}

Que la base de connaissances du lecteur joue un rôle décisif dans la compréhension écrite ne fait l'objet d'aucune contestation (Stahl \& Hiebert, 2005; Perfetti \& Stafura, 2014). Plus il connaît de choses sur le sujet traité, mieux il comprend. La relation inverse est également vraie : plus il lit, plus il apprend de mots et de connaissances (Stanovitch, 1986; Stanovich \& Cunnigham, 1993). Autrement dit, la relation entre connaissances et compréhension en lecture est logiquement réciproque. Dès lors, nous aurions pu être tentés d'introduire dans le modèle SVR les connaissances du monde dont disposent les élèves. Nous ne l'avons pas fait car, comme Adlof, Perfetti et Catts (2011), nous pensions que les connaissances ont le même effet sur le facteur $C L$ que sur le facteur L. II était donc inutile de les inclure dans la mesure où, pour tester ces deux composants, nous avions construit des épreuves évaluant le même type de connaissances, notamment celles relatives aux théories de l'esprit.

Nous avons aussi hésité à ajouter les compétences lexicales dans notre modèle parce qu'elles semblaient déjà intégrées dans l'évaluation de $\mathrm{CL}$ et de L. En effet, nos tests d'évaluation de la compréhension, à l'oral comme à l'écrit, reposaient sur des textes issus de la littérature de jeunesse qui présentaient de réelles difficultés lexicales. Nous étions sensibles aux arguments de Perfetti (2010) et de Wagner, Herrera, Spencer et alii (2014) qui ne jugeaient pas nécessaire d'intégrer le vocabulaire au modèle SVR puisqu'il intervenait déjà dans les deux composants du modèle en facilitant la compréhension du langage oral et le décodage. Mais, comme pour les jeunes enfants son rôle n'est pas de même nature dans les deux registres, nous avons fina-

base de connaissances. Chaque élève disposait d'une feuille comprenant 15 planches en couleur, 3 pour expliquer la consigne, 12 pour le test. Cette épreuve, réalisée en passation semi-collective (6 élèves maximum), a été chronométrée. Deux scores ont été calculés : $1^{\circ}$ le nombre de bonnes réponses (maximum 12) et $2^{\circ}$ le temps mis par l'élève pour réaliser l'épreuve (avec arrêt au bout de lement choisi de l'étudier séparément. Au début de l'apprentissage, le poids du lexique semble plus important sur le versant de la compréhension du langage que sur celui du décodage: des recherches ont mis en évidence une absence d'effet de fréquence ou de lexicalité en lecture à haute voix chez les apprentis-lecteurs qui ont massivement recours à la voie phonologique (Sprenger-Charolles, Siegel, Béchennec et al., 2003). Les études qui ont montré I'influence positive des connaissances lexicales sur le décodage portaient sur des lecteurs plus âgés et plus expérimentés que les nôtres (Ouellette, 2006; Nation, 2008, 2009; Nation $\&$ Cocksey, 2009). C'est pourquoi nous avons ajouté une variable lexicale à notre modèle et nous avons comparé les performances des faibles décodeurs et des décodeurs habiles pour tester I'hypothèse d'une importance lexicale croissante avec la maîtrise du décodage (modèle $n^{\circ} 2$, hypothèse 4 ).

\section{Synthèse : le modèle $n^{\circ} 2$, un modèle SVR-Texte étendu}

Dans notre deuxième modèle, nous avons logiquement ajouté quatre composants à ceux du premier : $\mathrm{Ph}$ pour caractériser les habiletés phonologiques, FI pour la fluence, VOC pour le vocabulaire passif et RNV pour le raisonnement non verbal. La nouvelle équation est donc la suivante $: \mathrm{L}=\mathrm{D} \times \mathrm{Ph} \times \mathrm{FI} \times \mathrm{CL} \times \mathrm{VOC} \times \mathrm{RNV}$. Dans ce modèle $n^{\circ} 2$, les définitions de $L$, $D$ et $C L$ restent inchangées. La variable phonologique Ph est, dans un second temps, subdivisée en deux : Ph1 pour les habiletés syllabiques et $\mathrm{Ph} 2$ pour les habiletés phonémiques.

Notre hypothèse générale est double. Nous prévoyons, d'une part, que le modèle $n^{\circ} 2$ aura un pouvoir explicatif plus fort que le modèle $n^{\circ} 1$, et d'autre part que les compétences relatives à la compréhension du langage (textes entendus et vocabulaire) et au raisonnement non verbal seront aussi influentes que les compétences dans le domaine de la maîtrise du code (décodage, fluence et phonologie) dès la fin du cours préparatoire (modèle $\mathrm{n}^{\circ} 2$, hypothèse générale).

\section{Troisième question : les différences entre bons et faibles décodeurs}

Les synthèses portant sur les lecteurs qui comprennent mal alors qu'ils décodent aisément montrent que le cas est rare en début d'apprentissage (Hulme \& Snowling, 2011) et qu'il est généralement associé à de sérieuses 
difficultés langagières à l'oral. De manière générale, dès que les élèves atteignent un niveau de décodage suffisant, ce sont les compétences en compréhension de l'écrit entendu qui déterminent le niveau de compréhension en lecture autonome (Oakhill, Cain \& Bryant, 2003). L'automatisation des traitements de bas niveau libère des ressources attentionnelles pour les traitements de haut niveau dont la qualité devient par conséquent déterminante pour expliquer la qualité de la compréhension en lecture autonome. Si ce niveau est atteint par un grand nombre d'élèves dès la fin du cours préparatoire, notre modèle devrait mettre en évidence un impact important des compétences en compréhension du langage (compréhension de textes entendus et vocabulaire) et en raisonnement non verbal, mais les résultats devraient être variables selon le niveau de décodage des élèves. En d'autres termes, la compréhension écrite ne devrait pas être expliquée par les mêmes facteurs : niveau en décodage et analyse phonologique chez les faibles décodeurs, capacités de compréhension du langage oral, niveau de vocabulaire et de raisonnement non verbal chez les bons décodeurs.

Cette prédiction est cohérente avec les résultats de Gentaz, Sprenger-Charolles et Theurel (2015) prouvant que les élèves qui comprennent mal à la fin du cours préparatoire sont surtout ceux qui décodent mal. Les analyses de régression que ces chercheurs ont réalisées font apparaître d'importantes différences entre les facteurs explicatifs de la compréhension en lecture autonome selon les compétences de décodage.

Si notre modèle $n^{\circ} 2$ est valide, on devrait retrouver des résultats allant dans le même sens et affiner les contributions respectives des variables que nous avons construites. Nous étudierons donc les performances en compréhension d'élèves inégalement habiles en décodage en créant deux groupes, l'un composé des meilleurs décodeurs de notre échantillon, l'autre des plus faibles après en avoir soustrait les non-décodeurs. Notre dernière hypothèse, conforme au modèle SVR classique, est donc que le poids du décodage ira en diminuant et que le poids de la compréhension du langage ira en augmentant avec l'amélioration de la qualité du décodage (modèle $n^{\circ} 2$, hypothèse 5 ).

\section{Méthodologie de la recherche}

Pour identifier les facteurs explicatifs des performances en lecture-compréhension à la fin du cours préparatoire, nous avons évalué les performances des élèves en lecture autonome $(\mathrm{L})$ et calculé la part de variance dont les compétences de décodage (D) et de compréhension de textes entendus $(\mathrm{CL})$ rendent compte lorsqu'elles sont évaluées au même moment mais indépendamment. Pour ce faire, nous avons étudié des corrélations (coefficient de Pearson) entre facteurs et élaboré des modèles de régressions multiples à l'aide des logiciels SAS 9.4 et STATA.

\section{Le choix des épreuves d'évaluation}

Nos choix d'épreuves ${ }^{3}$ ont été guidés par deux principes : 1) sélectionner ou élaborer des épreuves cohérentes avec nos hypothèses théoriques, ce qui impliquait de ne pas en rester à la compréhension littérale de phrases ou de bribes de textes, tant en lecture qu'en audition; 2) sélectionner des épreuves utilisées dans des études analogues par nos collègues francophones experts du domaine afin de comparer les résultats et cumuler les connaissances sur la maîtrise du français écrit.

En France, avant que nous entreprenions notre étude, seuls Gentaz et ses collègues avaient mis à l'épreuve le modèle SVR au cours préparatoire. Ils l'avaient fait en 2010-2011 dans la région lyonnaise auprès de 392 élèves (Gentaz, Sprenger-Charolles \& Theurel, 2015).

Pour évaluer le facteur $L$, ils avaient choisi une épreuve de compréhension de phrases n'excédant pas dix mots dont le vocabulaire est très simple mais dont la complexité, croissante, repose sur celle de la syntaxe (test syntaxico-sémantique EcoSSe; Lecocq, 1996). S'ils avaient aussi administré aux enfants un test de compréhension d'un texte très court (Gentaz, Sprenger-Charolles, Theurel et al., 2013), ils n'ont pas utilisé en 2015 les résultats de cette épreuve dans leurs calculs relatifs aux prédicteurs de la compréhension (Gentaz, Sprenger-Charolles \& Theurel, 2015).

Pour respecter les exigences théoriques du modèle SVR, ils avaient évalué la compréhension orale $(C L)$ à I'aide de tests analogues à ceux de $L$ : ils avaient utilisé un test de compréhension de phrases entendues présentant le même type de difficultés que celles des phrases lues (ECoSSe; Lecocq, 1996).

3 Toutes les épreuves d'évaluation sont consultables en ligne: $<$ http://ife.ens-lyon.fr/ife/recherche/lire-ecrire/livret-consignesevaluateur-juin-2013.pdf> pour le début de CP et <http://ife.enslyon.fr/ife/recherche/lire-ecrire/livret-consignes-evaluateurjuin-2014.pdf> pour la fin de CP (consultés le 2 février 2018). 
Pour évaluer $D$, ils avaient créé un sub-test de lecture de pseudo-mots et de mots familiers en temps limité (deux fois une minute), ce qui permettait d'évaluer la précision et la vitesse du décodage. Dans leur publication, ils ont réduit le décodage au seul traitement des pseudo-mots.

Nous avons jugé que leurs choix méthodologiques pour évaluer $L$ et $C L$ ne pouvaient que renforcer le poids du décodage puisqu'ils réduisaient $L$ et $C L$ à la seule compréhension de phrases. De ce fait, ils affaiblissaient les exigences de la compréhension écrite (L) puisque leurs épreuves n'exigeaient pas l'élaboration d'une cohérence textuelle, mobilisaient peu les référents culturels et sollicitaient peu les mécanismes inférentiels. C'est pourquoi, bien que réutilisant les modalités d'évaluation choisies par nos collègues pour $D$, nous n'avons pas repris leur protocole pour $L$ et $C L$. Nous avons construit deux épreuves originales, cohérentes avec notre définition du modèle SVR.

Pour $L$, nous avons conçu une épreuve basée sur un récit complexe permettant de vérifier la qualité d'une compréhension littérale et inférentielle (épreuve "La poule noire et ses trois poussins ${ }^{4}$ ). Dans une première partie, les enfants devaient commencer à lire un court récit pour ensuite représenter la situation initiale en sélectionnant et en positionnant les images de personnages sur un décor. Dans la seconde, après la lecture intégrale du texte, ils devaient répondre oralement à des questions portant sur l'implicite du texte, posées par l'évaluateur. Ce dernier n'apportait aucune aide ou feedback.

Pour $C$, afin de respecter les exigences théoriques du modèle, nous avons élaboré une tâche présentant le même type de difficultés que pour L, c'est-à-dire une épreuve basée sur l'écoute de deux récits issus de la

4 L'épreuve de «La poule noire et ses trois poussins » débute par l'observation d'un décor (15 secondes environ) puis la lecture autonome du début d'un récit à l'issue de laquelle l'enfant doit choisir parmi 14 images celles qui représentent les personnages cités (4) et les placer au bon endroit sur le décor. Dans un second temps, la seconde partie du récit est démasquée puis lue à son tour.

Partie 1 - Dans la cour de la maison, une poule noire promène ses trois poussins. Félix, un gros chat, est caché sous le banc et voudrait bien en manger un. Mais il a peur du chien à l'oreille coupée qui est assis devant la porte de la maison. Partie 2-Un peu plus tard, le chien s'en va dormir dans sa niche et Félix est très content. Heureusement pour les poussins, une petite fille qui a tout vu arrive et fait partir le chat.

L'enfant est alors interrogé oralement (6 questions littérales et inférentielles) : qu'est-ce que le chat Félix veut manger? Pourquoi, au début de l'histoire, le chat ne mange-t-il pas les poussins? Pourquoi le chat est-il content de voir partir le chien? Est-ce que le chat mange littérature de jeunesse posant des problèmes de compréhension inférentielle similaires à ceux de l'épreuve de lecture autonome (épreuve «Deux récits : Anatole et L'oiseau de pluie»). Questions et réponses étaient délivrées à l'oral lors d'une évaluation individuelle réalisée à la suite de l'audition des récits préalablement enregistrés.

Dans les deux cas, nous avons choisi des récits complets dont la compréhension exigeait des capacités de raisonnement, des traitements de l'implicite, des connaissances encyclopédiques, une certaine familiarité avec les structures narratives, des compétences syntaxiques et lexicales.

Sur le plan lexical, nous avons utilisé le même test que Gentaz, Sprenger-Charolles, Theurel et alii (2013), le volet «vocabulaire passif» du test standardisé de vocabulaire actif et passif de Deltour et Hupkens (1980), le TVAP 5-8

Pour évaluer les compétences relatives au code, nous avons utilisé les mêmes tests que ceux de nos collègues : le test de Liliane Sprenger-Charolles «Lecture à haute voix de mots familiers et de mots inventés en une minute ${ }^{6}$ et celui de Sprenger-Charolles et collaborateurs $(2005,2010)$ pour la phonologie (le subtest phonologique de la batterie EVALEC). Nous avons ajouté ceux de Billard, Lequette, Pouget et alii (2013) pour la fluence (lecture de mots en contexte).

Toutes les compétences enfantines ont été évaluées à la fin du CP, à l'exception du raisonnement non verbal (RNV) et du test lexical (TVAP) qui ont été testés au début de l'année de $\mathrm{CP}$, comme dans l'étude de Gentaz et ses collaborateurs (2013). Nos collègues psychologues jugent inutile d'administrer ce test une seconde fois en fin d'année dans la mesure où les résultats sont stables à cette échelle temporelle.

5 Ce test permet l'évaluation du vocabulaire passif : I'élève doit choisir parmi six images celle qui illustre le mieux un mot prononcé par l'évaluateur. Ce test permet d'évaluer la connaissance de 30 mots courants ou plus rares : 2 points étaient attribués si l'élève désignait l'image exacte, 1 point pour une image proche, 0 dans les autres cas (score maximum : 60 points).

6 Ce test nous a été fourni en 2012 par l'auteur que nous remercions vivement. II est consultable, page 29 , à l'adresse : <http://ife. ens-lyon.fr/ife/recherche/lire-ecrire/livret-consignes-evaluateurjuin-2014.pdf> (consulté le 2 février 2018). Le manuel de passation et de cotation des épreuves utilisées dans les études de Gentaz et collaborateurs $(2013,2015)$ a été édité par la FAPSE-UNIGE : <https:// www.unige.ch/fapse/sensori-moteur/files/8614/9309/9601/LivretEvaluerLectureGentazTheureISprenger-Charolles2017.pdf $>$ (consulté le 2 février 2018). 


\section{L'échantillon d'élèves}

Nous l'avons dit plus haut, notre étude prend appui sur les données recueillies à l'occasion de l'enquête Lire et Écrire au CP dirigée par Goigoux dont le rapport a été publié en 2016 (Goigoux, 2016). Nous disposions, à la fin du cours préparatoire, d'un échantillon de 2507 élèves répartis dans 131 classes implantées dans 13 académies différentes et dont les performances ont été évaluées individuellement (voir Goigoux, Jarlegan \& Piquée, 2015 pour une présentation de la méthodologie générale et Jarlegan, Piquée, Fontanieu et Goigoux dans ce volume pour une présentation des résultats d'ensemble). La composition sociale de cet échantillon était légèrement plus défavorisée que la population nationale de référence ${ }^{7}$; près de $30 \%$ des classes étaient implantées en éducation prioritaire.

Nous avons d'abord supprimé de l'échantillon 18 élèves pour lesquels nous n'avions pas tous les résultats, notamment ceux requis pour le modèle $n^{\circ} 2$. Nous en avons ensuite éliminé 96 autres auxquels, pour des raisons éthiques, nous n'avions pas proposé l'épreuve de lecture autonome car ils n'avaient pas réussi à lire au moins quinze mots sur soixante-cinq en deux minutes dans l'épreuve de lecture de mots familiers et de pseudo-mots ${ }^{8}$. Nous ne voulions pas leur demander de réaliser une tâche totalement hors de leur portée afin de ne pas les mettre inutilement en difficulté. Nous ne voulions pas non plus renforcer chez eux une conception erronée de la lecture : leur laisser croire par exemple que, lorsqu'on ne sait pas déchiffrer, on peut deviner le sens d'un texte à partir d'illustrations. L'échantillon étudié ici est donc composé de 2395 élèves, soit 95,5\% de l'échantillon initial.

Ce choix méthodologique affecte nos résultats en affaiblissant le pouvoir explicatif du modèle (perte d'environ $5 \%$ selon notre simulation ${ }^{9}$ ) par comparaison

7 Le milieu social d'origine a été défini à partir de la profession la plus élevée des deux parents : 19,5\% appartiennent à un milieu favorisé, $28,1 \%$ à un milieu intermédiaire et $51,4 \%$ à un milieu défavorisé. $71,7 \%$ des élèves parlent uniquement le français à la maison contre $6,7 \%$ qui ne le parlent pas et $21,6 \%$ qui parlent au moins deux langues à la maison, dont le français.

8 À noter que nous avons proposé la même épreuve au début et à la fin du CP (65 items) alors que nos collègues en avaient ajoutés pour atteindre 120 items à la fin du CP. À noter aussi que les scores sont calculés sur 75 points car 10 items comptent double en raison de règles positionnelles (par exemple, variation de la valeur de $\mathrm{g}$ devant a ou devant e).

9 Nous avons procédé à une simulation en attribuant une note de 1,5 sur 15 en compréhension aux élèves non décodeurs que nous avions éliminés parce qu'ils obtenaient moins de 15 points sur 75 aux recherches qui n'éliminent pas les élèves non décodeurs. Dans celles-ci, l'estimation du poids de D est biaisée car les élèves qui ne savent pas décoder obtiennent un score quasi nul en lecture-compréhension autonome. Mais notre exigence méthodologique accroît en revanche la fiabilité du modèle en éliminant le biais de l'effet plancher. Elle apporte ainsi de précieuses indications sur le poids du décodage dans la compréhension.

\section{Bons et faibles décodeurs}

Pour examiner comment évolue le poids des différents composants du modèle lorsque l'on distingue bons et faibles décodeurs, nous avons construit une nouvelle variable centrée et réduite qui tient compte à la fois des capacités d'identification des pseudo-mots et mots isolés d'une part, et des mots lus en contexte d'autre part.

Nous avons créé deux sous-groupes composés, pour l'un, des bons décodeurs (scores supérieurs à un écart-type) et, pour l'autre, des mauvais décodeurs (scores inférieurs à moins un écart-type), soit respectivement 343 et 431 élèves.

\section{Premiers résultats descriptifs}

\section{Statistiques descriptives}

Si l'on examine les différences entre les sous-groupes des bons et faibles décodeurs (voir tableau 1), on constate que, dans tous les domaines, les résultats des premiers sont supérieurs à ceux des seconds. En décodage bien sûr, par construction, mais aussi en raisonnement non verbal $(d=0,8)^{10}$ et dans le domaine de la compréhension du langage : en compréhension de textes entendus $(d=0,6)$, en vocabulaire $(d=0,7)$. Les mauvais décodeurs cumulent plusieurs types de faiblesses qui se combinent pour gêner leur compréhension des textes. Les analyses de régression qui vont suivre éclaireront ce résultat.

Les bons décodeurs cumulent des habiletés dans tous les domaines, ce qui leur permet de bien comprendre

à l'épreuve de décodage (1,5/15 était la moyenne de ceux qui leur étaient immédiatement supérieurs, ceux qui avaient obtenu entre 15 et 20 points en décodage). Dans ce cas, le $R 2$ du modèle $n^{\circ} 2$ gagnait plus de 5 points pour atteindre $47 \%$ de part de variance expliquée.

10 Le d de Cohen donne une indication de l'ampleur d'une différence de moyennes en la rapportant à l'écart-type de l'épreuve. 
Tableau 1. Données descriptives pour l'ensemble des mesures effectuées

\begin{tabular}{|c|c|c|c|c|}
\hline \multirow[t]{2}{*}{$\begin{array}{l}\text { Tests en fin de CP } \\
\text { (sauf RNV et VOC en début) }\end{array}$} & $\begin{array}{l}\text { Tous les élèves } \\
(\mathrm{N}=2395)\end{array}$ & $\begin{array}{l}\text { Bons décodeurs } \\
\qquad(N=343)\end{array}$ & $\begin{array}{l}\text { Faibles } \\
\text { décodeurs } \\
(\mathrm{N}=431)\end{array}$ & $\begin{array}{l}\text { Comparaison deux } \\
\text { sous-groupes }\end{array}$ \\
\hline & $\begin{array}{c}\text { Moyenne } \\
\text { (sd : écart-type) } \\
\text { (Mini-maxi) }\end{array}$ & $\begin{array}{c}\text { Moyenne } \\
\text { (sd : écart-type) }\end{array}$ & $\begin{array}{c}\text { Moyenne } \\
\text { (sd : écart-type) }\end{array}$ & $\begin{array}{l}\mathrm{p} \text { de Student } \\
\mathrm{d} \text { de Cohen }\end{array}$ \\
\hline $\begin{array}{l}\mathrm{L}: \text { compréhension en lecture } \\
\text { autonome (en } \% \text { de réussite) }\end{array}$ & $\begin{array}{l}54,20 \\
(27,33) \\
(0-15) \\
\end{array}$ & $\begin{array}{c}72,13 \\
(24,73)\end{array}$ & $\begin{array}{c}30,53 \\
(12,29)\end{array}$ & $\begin{array}{l}<0,0001 \\
d=1,5\end{array}$ \\
\hline $\begin{array}{l}\text { D : décodage de mots familiers et } \\
\text { de pseudo-mots en deux fois une } \\
\text { minute (score sur } 75 \text { ) }\end{array}$ & $\begin{array}{c}61,08 \\
(14,93) \\
16-75 \\
\end{array}$ & $\begin{array}{l}72,51 \\
(2,32)\end{array}$ & $\begin{array}{l}33,54 \\
(9,11)\end{array}$ & $\begin{array}{l}<0,0001 \\
d=2,6\end{array}$ \\
\hline $\begin{array}{l}\text { Ph : habiletés phonologiques } \\
\text { (suppression de syllabes et } \\
\text { phonèmes; en } \% \text { de réussite) }\end{array}$ & $\begin{array}{c}66,74 \\
(27,88) \\
(0-34)\end{array}$ & $\begin{array}{c}84,97 \\
(17,93)\end{array}$ & $\begin{array}{c}42,97 \\
(28,11)\end{array}$ & $\begin{array}{l}<0,0001 \\
d=1,5\end{array}$ \\
\hline $\begin{array}{l}\text { Fl : fluence en contexte (nombre } \\
\text { de mots correctement lus en une } \\
\text { minute dans un texte) }\end{array}$ & $\begin{array}{l}40,11 \\
(26,46) \\
(0-153)\end{array}$ & $\begin{array}{c}88,39 \\
(18,60)\end{array}$ & $\begin{array}{l}10,61 \\
(5,32)\end{array}$ & $\begin{array}{l}<0,0001 \\
d=2,9\end{array}$ \\
\hline $\begin{array}{l}\mathrm{CL}: \text { compréhension de textes } \\
\text { entendus (en } \% \text { de réussite) }\end{array}$ & $\begin{array}{c}57,10 \\
(27,80) \\
(0-10)\end{array}$ & $\begin{array}{c}65,70 \\
(27,35)\end{array}$ & $\begin{array}{c}49,70 \\
(28,83)\end{array}$ & $\begin{array}{l}<0,0001 \\
d=0,6\end{array}$ \\
\hline VOC : vocabulaire passif (TVAP) & $\begin{array}{l}37,31 \\
(7,75) \\
(0-55) \\
\end{array}$ & $\begin{array}{l}40,06 \\
(7,31)\end{array}$ & $\begin{array}{l}34,56 \\
(8,43)\end{array}$ & $\begin{array}{c}<0,0001 \\
d=0,7\end{array}$ \\
\hline $\begin{array}{l}\text { RNV : raisonnement non verbal } \\
\text { (en \% de réussite) }\end{array}$ & $\begin{array}{c}36,42 \\
(19,75) \\
(0-12)\end{array}$ & $\begin{array}{c}44,17 \\
(20,23)\end{array}$ & $\begin{array}{c}28,83 \\
(17,71)\end{array}$ & $\begin{array}{c}<0,0001 \\
d=0,8\end{array}$ \\
\hline
\end{tabular}

les textes qu'ils lisent de manière autonome : seuls $10 \%$ d'entre eux comprennent moins bien que la moyenne de l'échantillon.

À une exception près, celle du TVAP, nous ne disposons pas de normes statistiques pour nos épreuves en raison de l'absence de tests standardisés, en français, pour les variables étudiées. Gageons que nos épreuves seront reprises et que nos résultats contribueront à cette standardisation. Notons aussi que la norme lexicale établie par Deltour et Hupkens (1980) il y a 40 ans pour des enfants de 7 ans était égale à 53,6 $(s d=4,7)$. Or, dans notre étude, la moyenne est de 37,3 $(s d=7,8)$ et de $40,2(s d=7,4)$ dans l'étude réalisée dans la région lyonnaise par Gentaz et collaborateurs (2013), avec $42,1(s d=7,6)$ pour les bons décodeurs et 37,7 $(s d=7,0)$ pour les faibles décodeurs. La norme de Deltour et Hupkens est probablement à réactualiser.

Notre échantillon, sociologiquement plus défavorisé que la moyenne nationale, est donc sur le plan lexical légèrement plus faible que celui de Gentaz deurs) restent similaires. Comme on peut aussi le voir sur le tableau 1, notre échantillon obtient des résultats inférieurs à ceux des élèves lyonnais en lecture de pseudo-mots et de mots familiers isolés : $61,1(14,9)$ en deux fois une minute pour notre échantillon contre $67,7(14,1)$ pour l'échantillon de Gentaz $(28,1$ pour les pseudo-mots et 39,6 pour les mots familiers). Sur le plan phonologique en revanche, notre échantillon est légèrement plus performant que le lyonnais : $66,7 \%$ $(27,8)$ de réussite contre $62,2 \%(31,1)$.

\section{Corrélations}

La corrélation entre décodage (pseudo-mots et mots isolés) et fluence (mots lus en contexte) est forte $(0,65)$ sans être extrême ce qui indique que ces deux mesures se complètent sans se confondre, justifiant a posteriori la pertinence d'introduire les deux dans le modèle (voir tableau 2). Ce raisonnement est encore plus valide pour les compétences phonologiques au regard des compétences de décodage (corrélation moyenne à 0,48 ). 
De la même manière, les performances en compréhension de textes entendus ne sont que moyennement corrélées à celles en lexique $(0,48)$, ce qui valide aussi la pertinence de notre choix d'introduire les deux dans le modèle SVR.

Signalons enfin que certains psychologues parlent aussi de fluence pour désigner la vitesse et la précision de lecture de mots isolés ou de pseudo-mots (ici D). Pour notre part, nous avons réservé ce terme à la lecture de mots en contexte (FI) même si la contrainte temporelle s'applique de la même manière aux deux épreuves. Nous avons fait ce choix pour respecter I'usage habituel du mot fluence dans le monde francophone de l'enseignement (professeurs, formateurs et chercheurs en sciences de l'éducation). Dans cet univers, il est utilisé pour décrire la fluidité de la lecture à haute voix de phrases et de textes, ce qui est cohérent avec la définition du mot français évoquant l'action de couler et qui est moins adapté à la lecture hachée de mots en listes.

\section{Analyse de régression en réponse à la première question}

Nous avons centré et réduit les variables afin de pouvoir comparer leur poids respectif. Au terme d'une analyse de régression multiple, nous avons donc évalué l'influence des facteurs D et CL sur la compréhension en lecture $(\mathrm{L})$ (voir tableau 3 ).

Notre premier modèle SVR-Texte permet d'expliquer $37,5 \%$ de la variance de $L\left(R^{2}=0,375\right)$. Les coefficients qui expriment le poids de l'effet de chacune des variables sont égaux à 0,51 pour le décodage et à 0,38 pour la compréhension de textes entendus. Autrement dit, l'augmentation d'un écart-type de D s'accompagne d'une élévation de 0,51 écart-type de $L$ alors qu'une augmentation d'un écart-type de CL entraîne une augmentation de 0,38 écart-type de L. Les deux facteurs pèsent donc fortement sur la compréhension en lecture, mais la compréhension des textes entendus influe moins que le décodage (un quart de moins). Cette comparaison des coefficients est valide, car les deux variables ont été préalablement centrées et réduites.

Parallèlement à la vérification des propriétés des résidus des modèles de régression linéaire multiple (distribution, homoscédasticité11) et en raison de la nature de la variable compauto (variable discrète et non continue avec des scores compris entre 0 et 15 et un

11 L'homoscédasticité est une propriété fondamentale du modèle de la régression linéaire générale : elle est attestée lorsque la variance des erreurs stochastiques de la régression est la même pour chaque observation. 
Tableau 4. Modèle $n^{\circ} 2$ SVR-Texte étendu $\mathrm{L}=\mathrm{D} \times \mathrm{FI} \times \mathrm{Ph} \times \mathrm{CL} \times \mathrm{VOC} \times \mathrm{RNV}$ : analyse de régression multiple pour la variable dépendante compréhension en lecture autonome $(\mathrm{N}=2395)$

\begin{tabular}{l|c|c}
\hline Compétences évaluées & $\begin{array}{c}\text { Coefficients } B \text { du modèle } 2 \\
\left(\mathbf{R}^{2}=0,435\right)\end{array}$ & $\begin{array}{c}\text { Rappel du modèle } 1 \\
\left(\mathbf{R}^{2}=0,375\right)\end{array}$ \\
\hline $\begin{array}{l}\mathrm{D}: \text { décodage de mots familiers et de pseudo-mots } \\
\text { en temps limité }\end{array}$ & $0,314^{* * *}$ & 0,509 \\
\hline $\mathrm{Fl}:$ fluence (lecture de mots en contexte) & $0,132^{* * *}$ & $/$ \\
\hline $\mathrm{Ph}:$ habiletés phonologiques & $0,086^{* * *}$ & $/$ \\
\hline $\mathrm{CL}:$ compréhension de textes narratifs entendus & $0,253^{* * *}$ & $\mathbf{0 , 3 8 1}$ \\
\hline VOC : vocabulaire passif & $0,156^{* * *}$ & $/$ \\
\hline RNV : raisonnement non verbal & $0,118^{* * *}$ & $/$ \\
\hline
\end{tabular}

Légende : ${ }^{*}: p<0,05 ;^{* *}: p<0,01 ;{ }^{* *}: p<0,001$.

pas de 0,5 point, soit 31 valeurs de scores possibles), nous avons analysé les résultats d'une régression logistique modélisant une variable à deux modalités exprimant respectivement la réussite ou l'échec aux tests de compréhension autonome. Ces résultats ont confirmé la validité des modèles présentés et le poids de chacun des scores dans l'explication du score de compréhension autonome ${ }^{12}$.

\section{Commentaire}

Nos résultats confortent et affinent ceux du paradigme SVR : 1) les compétences en décodage et en compréhension de textes entendus apportent une contribution significative à la compréhension en lecture de texte; 2 ) à la fin de la première année de la scolarité élémentaire, la contribution du code est plus importante que celle de la compréhension de textes entendus. Ils indiquent cependant que les différences entre ces deux facteurs sont beaucoup moins grandes que dans les études classiques basées sur la compréhension de phrases dans lesquelles le poids du décodage est au moins le double de celui de la compréhension (Kendeou, Savage \& van den Broek, 2009; Kendeou, van den Broek, White et al., 2009). Ici le poids de la compréhension de textes entendus représente près des trois quarts du poids du décodage. L'hypothèse générale du modèle $\mathrm{n}^{\circ} 1$ est donc vérifiée.

Cette différence s'explique par les choix méthodologiques exposés plus haut pour l'évaluation de $L$ et

12 Nous remercions chaleureusement Valérie Fontanieu, statisticienne à I'Institut français de l'éducation (IFÉ, ENS de Lyon), qui a de CL basée sur des textes complexes et non sur des phrases. Elle ouvre des perspectives didactiques intéressantes si on a l'ambition que les élèves étudient et comprennent tôt des textes cohérents et complexes, adaptés à leur âge. Elle révèle aussi l'importance de la contribution des compétences de compréhension de textes entendus et peut conduire à s'interroger sur les conditions didactiques à mettre en œuvre pour favoriser leur développement.

\section{Analyse de régression en réponse à la deuxième question}

Au terme d'une seconde analyse de régression multiple, nous avons évalué l'influence des facteurs D, Fl, Ph, $\mathrm{CL}, \mathrm{VOC}$ et RNV sur la compréhension en lecture $(\mathrm{L})$ à la fin du cours préparatoire (voir tableau 4). Ce second modèle permet d'expliquer $43,5 \%$ de la variance de $L$ soit une augmentation de 6 points par rapport au modèle $n^{\circ} 1$. Il semble donc plus pertinent. L'hypothèse générale du modèle $\mathrm{n}^{\circ} 2$ est donc vérifiée.

Le coefficient qui exprime le poids de l'effet de la variable décodage est égal à 0,314 pour le décodage, c'est-à-dire nettement moins que dans le modèle $n^{\circ} 1$, probablement sous l'influence de l'introduction des facteurs phonologiques et de fluence qui évaluent des compétences partiellement corrélées avec le décodage (voir tableau 2) et dont les poids sont importants (coefficients de 0,086 et 0,132). Les hypothèses 1 et 2 du modèle $n^{\circ} 2$ sont vérifiées.

L'introduction dans le modèle des scores aux tests de vocabulaire et de raisonnement non verbal (contributions importantes : 0,156 et 0,118 ) s'accompagne 
Tableau 5. Modèle $\mathrm{n}^{\circ} 3$ SVR-Texte étendu $\mathrm{L}=$ Dpseudo $x$ Dfam $x$ Phsyll $x$ Phphon $x$ FI $x$ CL $x$ VOC $x$ RNV : analyse de régression multiple pour la variable dépendante compréhension en lecture autonome $(\mathrm{N}=2395)$

\begin{tabular}{|c|c|c|c|}
\hline Compétences évaluées & Nouvelles variables & $\begin{array}{c}\text { Coefficients } B \\
\text { du modèle } 3 \\
\left(R^{2}=0,435\right)\end{array}$ & $\begin{array}{c}\text { Rappel du } \\
\text { modèle } 2 \\
\left(R^{2}=0,435\right)\end{array}$ \\
\hline \multirow{2}{*}{$\begin{array}{l}\text { D : décodage de mots familiers et de } \\
\text { pseudo-mots en temps limité }\end{array}$} & Dpseudo: décodage pseudo-mots & $0,136^{* * *}$ & \multirow[t]{2}{*}{$0,314^{* * *}$} \\
\hline & Dfam : décodage mots familiers & $0,185^{* * *}$ & \\
\hline \multirow[t]{2}{*}{$\mathrm{Ph}$ : habiletés phonologiques } & Phsyll : suppression syllabes & $0,024^{*}$ & \multirow[t]{2}{*}{$0,086^{* * *}$} \\
\hline & Phphon : suppression phonèmes & $0,070^{* *}$ & \\
\hline $\begin{array}{l}\mathrm{Fl} \text { : fluence (lecture de mots en } \\
\text { contexte) }\end{array}$ & inchangée & $0,133^{* * *}$ & $0,132^{* * *}$ \\
\hline $\begin{array}{l}\mathrm{CL}: \text { compréhension de textes } \\
\text { entendus }\end{array}$ & inchangée & $0,253^{* * *}$ & $0,253^{* * *}$ \\
\hline VOC : vocabulaire passif & inchangée & $0,156^{* * *}$ & $0,156^{* * *}$ \\
\hline RNV : raisonnement non verbal & inchangée & $0,118^{* * *}$ & $0,118^{* * *}$ \\
\hline
\end{tabular}

Légende : ${ }^{*}: p<0,05 ;{ }^{* *}: p<0,01 ;{ }^{* *}: p<0,001$.

d'une diminution du facteur $C L$ (compréhension de texte entendus) qui reste cependant à un niveau élevé $(0,253)$, proche de $D$. Les hypothèses 3 et 4 du modèle $\mathrm{n}^{\circ} 2$ sont aussi vérifiées.

Si l'on scinde les compétences en deux blocs et si I'on compare le poids de celles liées aux traitements de bas niveau $(\mathrm{D}=0,31, \mathrm{FI}=0,13$ et $\mathrm{Ph}=0,09)$ à celui des compétences liées aux traitements de haut niveau $(C L=0,25, \operatorname{VOC}=0,16$ et $\mathrm{RNV}=0,12)$, on constate qu'ils sont équivalents à la fin du CP.

Ces résultats confirment l'analyse proposée dans le cadre du modèle $n^{\circ} 1$ et l'affinent. Ils attestent l'importance de l'automatisation des processus d'identification des mots (vitesse et précision) et des compétences phonologiques, incitant les enseignants à leur apporter un soin particulier au cours préparatoire. S'ils permettent aussi de souligner l'importance du vocabulaire et des capacités de raisonnement, ils confirment surtout le poids important de la compétence à comprendre les textes entendus qui mobilise, outre des capacités de raisonnement et des connaissances lexicales, des connaissances textuelles et encyclopédiques déterminantes.

Nos résultats confortent également les conclusions de l'équipe de Gentaz qui a comparé l'influence du raisonnement non verbal sur une épreuve de compréhension de textes et sur une épreuve de compréhension de phrases. Elle est plus forte dans le premier cas que dans le second ce qui renforce la validité de notre modèle $n^{\circ} 1$ SVR-Texte. Rappelons que ces deux épreuves ne mesurent pas les mêmes composantes de la compréhension écrite (Keenan \& Betjemann, 2006). Aussi, si l'on veut s'approcher des exigences d'une authentique lecture autonome, est-il souhaitable que les recherches futures privilégient l'évaluation de la compréhension inférentielle de texte.

Cette conclusion est sans doute la contribution la plus originale et la plus importante de notre étude. Elle vient à point nommé pour étayer le nouveau programme du cycle 2 (MENESR, 2015) qui préconise un enseignement explicite de la compréhension de textes entendus au cours préparatoire, sans attendre que les élèves maitrisent le décodage.

\section{Investigations complémentaires}

L'utilisation de quatre subtests proposés par Sprenger-Charolles pour évaluer $D$ et $P h$ nous a permis d'étudier, d'une part, la répartition des contributions de la lecture des pseudo-mots et des mots familiers en D, et, d'autre part, celle des contributions des habiletés phonologiques syllabiques et phonémiques en $\mathrm{Ph}$ (voir le modèle $n^{\circ} 3$ dans le tableau 5 ).

Dans la mesure où les subtests distingués dans le modèle $n^{\circ} 3$ sont déjà présents dans le modèle $n^{\circ} 2$, le pouvoir explicatif des deux modèles est le même : $43,5 \%$. Seule la répartition des sous-scores, dont la somme égale ceux du score unique, procure une information complémentaire. C'est ainsi que nous pouvons constater que, à la fin du cours préparatoire, les compétences en lecture de mots familiers sont plus discriminantes que celles en lecture de pseudo-mots $(0,185$ 
Tableau 6. Modèle $\mathrm{n}^{\circ} 4$ SVR-Texte étendu $\mathrm{L}=\mathrm{D} \times \mathrm{Ph} \times \mathrm{FI} \times \mathrm{CL} \times \mathrm{VOC} \times \mathrm{RNV}$ : deux analyses de régression multiple pour la variable dépendante compréhension en lecture autonome réalisées pour les bons décodeurs $(\mathrm{N}=343$ ) et les mauvais décodeurs ( $\mathrm{N}=431$ élèves) avec rappel de l'ensemble de la population $(\mathrm{N}=2395)$

\begin{tabular}{|c|c|c|c|}
\hline Compétences évaluées & $\begin{array}{c}\text { Coefficients } B \text { du } \\
\text { modèle } 4 \text { pour les } \\
\text { bons décodeurs } \\
\left(R^{2}=0,305\right)\end{array}$ & $\begin{array}{l}\text { Coefficients } B \text { du } \\
\text { modèle } 4 \text { pour les } \\
\text { mauvais décodeurs } \\
\quad\left(R^{2}=0,260\right)\end{array}$ & $\begin{array}{c}\text { Rappel des } \\
\text { coefficients B pour } \\
\text { l'ensemble } \\
\left(R^{2}=0,435\right)\end{array}$ \\
\hline $\begin{array}{l}\text { D : décodage de mots familiers et de pseu- } \\
\text { do-mots (en temps limité) }\end{array}$ & 0,180 (ns) & $0,339 * * *$ & $0,314^{* * *}$ \\
\hline $\mathrm{Ph}$ : habiletés phonologiques & 0,034 (ns) & $0,136^{* * *}$ & $0,086^{* * *}$ \\
\hline $\begin{array}{l}\text { Fl : fluence (lecture de mots en contexte en } \\
\text { temps limité) }\end{array}$ & 0,098 (ns) & 0,222 (ns) & $0,132^{* * *}$ \\
\hline $\mathrm{CL}$ : compréhension de textes entendus & $0,237^{* * *}$ & $0,191^{* * *}$ & $0,253^{* * *}$ \\
\hline VOC : vocabulaire passif & $0,199 * *$ & $0,073^{*}$ & $0,156^{* * *}$ \\
\hline \multirow[t]{2}{*}{ RNV : raisonnement non verbal } & $0,212^{* * *}$ & $-0,022$ (ns) & $0,118^{* * *}$ \\
\hline & $N=343$ & $N=431$ & $N=2395$ \\
\hline
\end{tabular}

Légende $*^{*}: p<0,05 ;^{* *}: p<0,01 ;^{* * *}: p<0,001$.

contre 0,136). Ce résultat valide a posteriori notre choix d'examiner les unes et les autres et pas seulement la lecture de pseudo-mots.

Sur le plan phonologique, l'épreuve de suppression de phonèmes est plus discriminante que celle portant sur la suppression de syllabes $(0,070$ contre $0,024)$ ce qui est conforme aux résultats de Gentaz, Sprenger-Charolles et leurs collaborateurs (2013 et 2015) et à ceux de la méta-analyse de Melby-Lervag, Lyster et Hulme (2012) : ce sont surtout les capacités d'analyse phonémique qui sont déterminantes pour les compétences en lecture.

\section{Résultats en réponse à la troisième question}

Nous avons fait l'hypothèse (hypothèse 5 du modèle $\mathrm{n}^{\circ} 2$ ) que le poids du décodage devrait diminuer et celui de la compréhension du langage augmenter avec I'amélioration de la qualité du décodage. On trouve dans le tableau 6 les analyses des données qui permettent de la valider.

\section{Bons et mauvais décodeurs}

Nous constatons que, pour expliquer la capacité à comprendre un texte narratif en lecture autonome, le poids des habiletés de bas niveau ( $D$, Ph et FL) est beaucoup correspondants à ces habiletés sont non significatifs chez les bons décodeurs. Pour les faibles décodeurs, c'est le poids du déchiffrage de pseudo-mots et de mots isolés qui est le principal prédicteur, la fluence de la lecture de mots en contexte est non significative. Pour les bons décodeurs, ce sont les habiletés de haut niveau (CL, VOC et RNV) qui font la différence.

En d'autres termes, les analyses de régression font apparaître d'importantes différences entre les prédicteurs de la compréhension selon les compétences de décodage des jeunes élèves : chez les bons décodeurs, cette compréhension s'explique essentiellement par des compétences langagières au niveau lexical et supra-lexical et par des compétences de raisonnement alors que, chez les faibles décodeurs, elle s'explique surtout par le décodage et les capacités d'analyse phonémique.

\section{Des bons lecteurs mauvais décodeurs?}

Pour terminer, nous avons inventorié le nombre de sujets qui réussissent l'épreuve de compréhension en lecture autonome malgré de faibles performances en décodage (Cain \& Oakhill, 2006) et nous avons examiné si notre échantillon comptait aussi peu d'élèves «bons compreneurs - mauvais décodeurs» que dans l'étude lyonnaise menée par l'équipe de Gentaz. Pour les identifier, nous avons sélectionné les scores «hors normes» au sens gaussien du terme, c'est-à-dire ceux qui se situaient au-delà et en deçà des seuils de plus ou moins 
un écart-type. Nous avons donc appelé «bons compreneurs» les élèves dont le score à l'épreuve compauto (compréhension autonome en lecture de texte narratif) était supérieur à un écart-type : nous avons identifié 507 élèves dans ce cas qui avaient obtenu plus de 12 points sur 15 à cette épreuve. Nous avons appelé "mauvais décodeurs» ceux dont le score Impmf (lecture de pseudo-mots et de mots familiers en temps limité) était inférieur à moins un écart-type : nous avons ainsi identifié 332 élèves obtenant moins de 42 points sur 75 à cette épreuve. Parmi ces élèves, seuls 9 étaient à la fois «mauvais décodeurs » et «bons compreneurs», soit 0,4\% de l'échantillon. À noter que 7 sur les 9 étaient d'excellents compreneurs de textes entendus (leur score comptxtfz était supérieur à $+1,2$ écarttype) et pouvaient compenser ainsi une partie de leurs carences en décodage.

Ce résultat conforte, à une plus grande échelle, ceux de Gentaz, Spenger-Charolles, Theurel et alii $(2013,2015)$ : à la fin du cours préparatoire, il est exceptionnel qu'un élève comprenne un texte lu sans maîtriser le décodage. Et presque tous les bons décodeurs comprennent ce qu'ils lisent mais il faut souligner qu'ils sont aussi les élèves les plus performants en compréhension de textes entendus. Autrement dit, cette compétence de décodage, si elle n'est pas suffisante, est absolument nécessaire.

\section{Conclusion}

L'étude que nous venons de présenter aboutit à des résultats légèrement différents de ceux obtenus par l'équipe de Gentaz ${ }^{13}$ en raison de choix méthodologiques différents sur trois points : la composition de l'échantillon (392 élèves scolarisés en éducation prioritaire alors que notre échantillon, six fois plus grand, était plus représentatif de la population française); la nonprise en compte des élèves non déchiffreurs en fin de CP dans notre étude; et, surtout, le choix des épreuves retenues pour évaluer la compréhension privilégiant la compréhension de phrases dans l'étude lyonnaise et la compréhension de textes lus et entendus pour nous.

13 Dans la publication de 2015 , le modèle explique $58 \%$ de la variance de L. Les coefficients qui expriment le poids de chacune des variables sont égaux à 0,46 pour le décodage, à 0,17 pour la conscience phonémique, à 0,07 pour la conscience syllabique, à 0,22 pour la compréhension de phrases entendues et à 0,16 pour les compétences lexicales. Le poids du quotient intellectuel non verbal testé par l'épreuve de Raven $(0,10)$ est statistiquement non significatif.
Malgré ces différences, nos deux études apportent des informations qui vont dans le même sens et confortent les modèles SVR étendus. Les facteurs expliquant la compréhension écrite en fin de première année du primaire sont le décodage, la compréhension du langage (phrases ou textes entendus) et le vocabulaire. Le poids du premier facteur est plus fort que celui des deux autres mais l'écart est minime dès la fin du cours préparatoire pour une majorité d'élèves. Pour les meilleurs décodeurs, le poids de la compréhension du langage est supérieur à celui du décodage. Lorsque l'évaluation de la compréhension en lecture autonome porte sur des textes comportant une forte dose d'implicite, le facteur raisonnement non verbal joue aussi un rôle important, supérieur à celui des compétences phonologiques dont le pouvoir explicatif, bien que faible, est significatif surtout au niveau phonémique.

Nos résultats attestent l'importance des compétences autres que celles de décodage, en sus de celles-ci, et peuvent servir d'arguments pour encourager les enseignants à ne pas les négliger au début de l'apprentissage de la lecture. II est légitime de recommander de ne pas tout miser sur le décodage et de commencer tôt un enseignement explicite de la compréhension dès lors qu'il s'appuie sur des textes lus à haute voix aux enfants. Au vu de nos résultats, il ne semble donc plus possible d'invoquer le modèle SVR pour légitimer la faible part accordée à l'enseignement de la compréhension au cours préparatoire mais on peut le faire au contraire pour promouvoir un enseignement précoce de la compréhension de textes entendus (van den Broek, Kendeou, Lousberg et al., 2011 ; Bianco, 2015; Cèbe, 2016).

Roland Goigoux Université Clermont-Auvergne, ACTé (EA 4281) roland.goigoux@uca.fr

Sylvie Cèbe Université Clermont-Auvergne, ACTé (EA 4281) sylvie.cebe@uca.fr

Julie Pironom Université Clermont-Auvergne, ACTé (EA 4281) julie.pironom@uca.fr 


\section{Bibliographie}

ADLOF S. M., CATTS H. W. \& LITTLE T. D. (2006). «Should the simple view of reading include a fluency component?». Reading and Writing, $\mathrm{n}^{\circ}$ 19(9), p. 933-958.

ADLOF S. M., PERFETTI C. A. \& CATTS H. W. (2011). «Developmental changes in reading comprehension : implications for assessment and instruction». In S. J. Samuels \& A. E. Farstrup (dir.), What research has to say about reading instruction. Newark : International Reading Association, p.188-214.

BIANCO M. (2010). «La compréhension de textes : peut-on l'apprendre et l'enseigner?». In M. Crahay \& M. Dutrevis (dir.), Psychologie des apprentissages scolaires. Bruxelles : De Boeck, p.229-256.

BIANCO M. (2015). Du langage oral à la compréhension de l'écrit. Grenoble : Presses universitaires de Grenoble.

BILLARD C., LEQUETTE C., POUGET G., POURCHET M. \& ZORMAN M. (2013). OURA LEC - CP. Outil de repérage des acquis en lecture des élèves en CP à l'usage des enseignants. En ligne : <http://www.cognisciences.com/IMG/OUTILSTEST.pdf> (consulté le 2 février 2018).

BRONCKART J.-P., BAIN D. E., SCHNEUWLY B., DAVAUD C. \& PASQUIER A. (1985). Le fonctionnement des discours : un modèle psychologique et une méthode d'analyse. Neuchâtel : Delachaux \& Niestlé.

CAIN K. \& OAKHILL J. (2006). «Profiles of children with specific reading comprehension difficulties». British Journal of Educational Psychology, n 76, p. 683-696.

CÈBE S. (2016). Quelles sont les compétences requises pour comprendre un texte écrit et comment les enseigner? Note de synthèse préparatoire à la conférence de consensus «Lire, comprendre, apprendre : comment soutenir le développement de compétences en lecture?». En ligne: <http://www. cnesco.fr/fr/lecture/paroles-dexperts/pratiques-enseignantes/> (consulté le 2 février 2018).

CNESCO (2016). Conférence de consensus: «Lire, comprendre, apprendre : comment soutenir le développement de compétences en lecture?». En ligne : <http://www.cnesco.fr/ lecture/> (consulté le 2 février 2018).

COLÉ P., CASALIS V., BELEN DOMINGUEZ A., LEYBAERT J., SCHELSTRAETE M A. \& SPRENGER-CHAROLLES L. (2012). Lecture et pathologies du langage oral. Grenoble : Presses universitaires de Grenoble.

COMPTON D. L., FUCHS D., FUCHS L. S., ELLEMAN A. M. \& GILBERT J. K. (2008). «Tracking children who fly below the radar: Latent transition modeling of students with late-emerging reading disability ». Learning and Individual Differences, ${ }^{\circ} 18(3)$, p. 329-337.

CUTTING L. E., MATEREK A., COLE C. A., LEVINE T. M. \& MAHONE E. M. (2009). «Effects of fluency, oral language, and executive function on reading comprehension performance». Annals of dyslexia, n59(1), p.34-54.

DEHAENE S. (dir.) (2011). Apprendre à lire. Des sciences cognitives à la salle de classe. Paris: Odile Jacob. et passif pour enfants de 5 à 8 ans (TVAP 5-8). Braine-leChâteau : Éd. de l'Application des Techniques Modernes.

FLORIT E., ROCH M. \& LEVORATO M. C. (2011). «Listening text comprehension of explicit and implicit information in preschoolers: The role of verbal and inferential skills». Discourse Processes, $\mathrm{n}^{\circ}$ 48(2), p. 119-138.

FUCHS D. \& YOUNG C. L. (2006). «On the irrelevance of intelligence in predicting responsiveness to reading instruction ». Exceptional Children, $\mathrm{n}^{\circ} 73, \mathrm{p} .8-30$.

GENTAZ E., SPRENGER-CHAROLLES L., THEUREL A. \& GURGAN M. (2013). «Évaluation quantitative d'un entraînement à la lecture à grande échelle pour des enfants de CP scolarisés en réseaux d'éducation prioritaire : apports et limites». ANAÉ, n ${ }^{\circ} 23$, p. 172-181.

GENTAZ E., SPRENGER-CHAROLLES L., THEUREL A. \& COLÉ P. (2013). «Reading Comprehension in a Large Cohort of French First Graders from Low Socio-Economic Status Families: A 7-Month Longitudinal Study». PLOS ONE, $\mathrm{n}^{\circ} 8(11)$. En ligne : <http://journals.plos.org/plosone/ article?id=10.1371/journal.pone.0078608> (consulté le 2 février 2018).

GENTAZ E., SPRENGER-CHAROLLES L. \& THEUREL A. (2015). «Differences in the Predictors of Reading Comprehension in First Graders from Low Socio-Economic Status Families with Either Good or Poor Decoding Skills». PloS ONE, $\mathrm{n}^{\circ}$ 10(3). En ligne : <http://journals.plos.org/plosone/ article?id=10.1371/journal.pone.0119581> (consulté le 2 février 2018).

GERSNBACHER M. A., VARNER K.R. \& FAUST M. (1990). «Investigating differences in general comprehension skill ». Journal of expérimental psychology : learning, memory and cognition, $\mathrm{n}^{\circ} 16$, p. 430-445.

GOIGOUX R. (dir.) (2016). «Étude de l'influence des pratiques d'enseignement de la lecture et de l'écriture sur la qualité des premiers apprentissages ». Rapport de recherche remis à Madame la directrice générale de l'enseignement scolaire (DGESCO-MENESR), ministère de l'Éducation nationale, de I'Enseignement supérieur et de la Recherche. En ligne : $<$ http://ife.ens-lyon.fr/ife/recherche/lire-ecrire> (consulté le 2 février 2018).

GOIGOUX R., JARLÉGAN A. \& PIQUÉE C. (2015). «Évaluer l'influence des pratiques d'enseignement du lire-écrire sur les apprentissages des élèves : enjeux et choix méthodologiques». Recherches en didactiques, $\mathrm{n}^{\circ} 19, \mathrm{p} .33-52$.

GOUGH P. B. \& TUNMER W. E. (1986). «Decoding, reading, and reading disability». Remedial and special education, $\mathrm{n}^{\circ} 7(1)$, p. 6-10.

HOOVER W. A. \& GOUGH P. B. (1990). «The simple view of reading ». Reading and Writing: An Interdisciplinary Journal, $\mathrm{n}^{\circ} 2, \mathrm{p} .127-160$.

HULME C. \& SNOWLING M. J. (2011). «Children's reading comprehension difficulties: Nature, causes, and treatments ». Current Directions in Psychological Science, $n^{\circ} 20$ (3), p. 139-142. 
KEENAN J. M. \& BETJEMANN R. S. (2006). «Comprehending the gray oral reading test without reading it: Why comprehension tests should not include passage-independent items ». Scientific Studies of Reading, $\mathrm{n}^{\circ} 10$, p.363-380.

KEENAN J. M., BETJEMANN R. S. \& OLSON R. K. (2008). « Reading comprehension tests vary in the skills they assess: Differential dependence on decoding and oral comprehension». Scientific Studies of Reading, vol.12, $\mathrm{n}^{\circ} 3$, p.281-300.

KEENAN J. M., OLSON R. K. \& BETJEMANN R. S. (2009). "Assessment and Etiolgy of Individual Differences in Reading Comprehension ». In R. K. Wagner, C. Schatschneider \& C. Phytian-Sence (dir.), Beyond Decoding. New York: The Guilford Press, p.227-245.

KENDEOU P., SAVAGE R. \& VAN DEN BROEK P. (2009). «Revisiting the simple view of reading ». British Journal of Educational Psychology, n 79 (2), p. 353-370.

KENDEOU P., VAN DEN BROEK P., WHITE M. J. \& LYNCH J. S. (2009). «Predicting reading comprehension in early elementary school: The independent contributions of oral language and decoding skills ». Journal of Educational Psychology, $n^{\circ} 101(4)$, p.765-778.

KHOMSI A. (1992). Perceptions, analogies et manipulations spatiales: PAMS. Montreuil : Éditions du Centre de psychologie appliquée (ECPA).

LECOCQ P. (1996). E.C.O.S.SE : une épreuve de compréhension syntaxico-sémantique. Lille : Presses du Septentrion.

MELBY-LERVAG M., LYSTER S. A. \& HULME C. (2012). «Phonological skills and their role in learning to read: a meta-analytic review». Psychological Bulletin, vol 138(2), p.322-352.

MEGHERBI H., SEIGNEURIC A. \& EHRLICH M. F. (2006). «Reading comprehension in French 1st and 2nd grade children: Contribution of decoding and language comprehension ". European Journal of Psychology of Education, vol. XXI, $n^{\circ} 2$, p.135-147.

MINISTÈRE DE L'ÉDUCATION NATIONALE [MEN] (1992). La maîtrise de la langue à l'école. Paris: CNDP/Savoir Livre.

MINISTÈRE DE L'ÉDUCATION NATIONALE DE L'ENSEIGNEMENT SUPÉRIEUR ET DE LA RECHERCHE [MENESR] (2015). «Programme de l'école élémentaire». Bulletin officiel spécial, $\mathrm{n}^{\circ} 11$ du 26 novembre 2015. En ligne : <http://www.education.gouv.fr/cid95812/au-bo-special-du-26-novembre2015-programmes-d-enseignement-de-I-ecole-elementaire-et-du-college.html> (consulté le 2 février 2018).

MINISTÈRE DE L'ÉDUCATION NATIONALE DE L'ENSEIGNEMENT SUPÉRIEUR ET DE LA RECHERCHE [MENESR] (2016). Ressources d'accompagnement du programme du cycle 2 . En ligne : <http://eduscol.education.fr/pid34140/francais. html> (consulté le 2 février 2018).

MUTER V., HULME C., SNOWLING M. \& STEVENSON J. (2004). "Phonemes, rimes, vocabulary, and grammatical skills as foundations of early reading development: Evidence from a longitudinal study ». Developmental Psychology, vol.40, $\mathrm{n}^{\circ} 5$, p. 665-681.
NATION K. (2008). «Learning to read words». The Quarterly Journal of Experimental Psychology, $\mathrm{n}^{\circ} 61(8), \mathrm{p} .1121-1133$.

NATION K. (2009). «Reading comprehension and vocabulary». In R. K. Wagner, C. Schatschneider \& C. Phythian-Sence (dir.), Beyond decoding. The behavioral and biological foundations of reading comprehension. New York : The Guilford Press, p.176-194.

NATION K. \& COCKSEY J. (2009). «The relationship between knowing a word and reading it aloud in children's word reading development ». Journal of experimental child psychology, $\mathrm{n}^{\circ}$ 103(3), p.296-308.

NATION K., COCKSEY J., TAYLOR J. S. H. \& BISHOP D. V. M. (2010). «A longitudinal investigation of early reading and language skills in children with poor reading comprehension ». Journal of Child Psychology and Psychiatry, n 51(9), p.1031-1039.

OAKHILL J. \& CAIN K. (2012). «The precursors of reading ability in young readers: Evidence from a four-year longitudinal study ». Scientific Studies of Reading, n 16, p. 91-121.

OAKHILL J., CAIN K. \& ELBRO C. (2014). Understanding and teaching reading comprehension: a handbook. Londres : Routledge.

OAKHILL J., CAIN K. \& BRYANT P. (2003). «The dissociation of word reading and text comprehension: Evidence from component skills». Language and Cognitive Processes, vol. $18, n^{\circ} 4$, p. 443-468.

OBSERVATOIRE NATIONAL DE LA LECTURE [ONL] (1998). Apprendre à lire. Paris : Odile Jacob.

OUELLETTE G. P. (2006). «What's meaning got to do with it: The role of vocabulary in word reading and reading comprehension ». Journal of educational psychology, $\mathrm{n}^{\circ}$ 98(3), p.554-566.

OUELLETTE G. \& BEERS A. (2010). «A not-so-simple view of reading: How oral vocabulary and visual-word recognition complicate the story». Reading and Writing, $\mathrm{n}^{\circ} 23$, p.189-208.

PERFETTI C. A., LANDI N. \& OAKHILL J. (2005). «The acquisition of reading comprehension skill ». In M. J. Snowling \& C. Hulmes (dir.), The science of reading: a handbook. Oxford : Blackwell, p.227-247.

PERFETTI C. (2010). «Decoding, vocabulary, and comprehension: the golden triangle of reading skill». In M. G. McKeown \& L. Kucan (dir.), Bringing reading research to life. New York : The Guilford Press, p.291-303.

PERFETTI C. \& STAFURA J. (2014). «Word knowledge in a theory of reading comprehension ». Scientific Studies of Reading, $\mathrm{n}^{\circ} 18(1)$, p. 22-37.

PINNELL G. S. (1995). «Listening to children read aloud: data from NAEP's integrated reading performance record (IRPR) at grade». Washington : The Nation Report Card. En ligne: <http://files.eric.ed.gov/fulltext/ED378550.pdf> (consulté le 2 février 2018).

PROGRAMME INCITATIF DE RECHERCHE EN ÉDUCATION ET FORMATION [PIREF] (2003). L'enseignement de la lecture à l'école primaire. Des premiers apprentissages au lecteur compétent. Conférence de consensus organisée à Paris les 4 
et 5 décembre 2003. En ligne: $<$ http://www.cndp.fr/bienlire/01-actualite/c-en-parle06.asp> (consulté le 2 février 2018).

SPRENGER-CHAROLLES L., SIEGEL L. S., BÉCHENNEC D. \& SERNICLAES W. (2003). «Development of phonological and orthographic processing in reading aloud, in silent reading, and in spelling: A four-year longitudinal study ». Journal of Experimental Child Psychology, n84(3), p.194-217.

SPRENGER-CHAROLLES L., COLÉ P., BÉCHENNEC D. \& KIPFFER-PIQUARD A. (2005). «French normative data on reading and related skills from EVALEC, a new computerized battery of tests ». European Review of Applied Psychology, $\mathrm{n}^{\circ} 55,157-186$.

SPRENGER-CHAROLLES L., COLÉ P., PIQUARD-KIPFFER A. \& LELOUP G. (2010). EVALEC enfant, Batterie informatisée d'évaluation diagnostique des troubles spécifiques d'apprentissage de la lecture. Paris : Ortho éditions.

SPRENGER-CHAROLLES L. (2013). «Principaux facteurs expliquant la réussite et l'échec de l'apprentissage de la lecture». ANAÉ, vol.24, $\mathrm{n}^{\circ} 116, \mathrm{p} .10-18$.

SPRENGER-CHAROLLES L. \& COLÉ P. (2013). Lecture et dyslexie. Approche cognitive. Paris : Dunod.

STAHL S. A. \& HIEBERT E. H. (2005). «The "word factors": A problem for reading comprehension assessment». In S. G. Paris \& S. A. Stahl, Children's reading comprehension and assessment. Londres : Erlbaum, p.161-186.

STANOVICH K. E. (1986). « Matthew effects in reading: Some consequences of individual differences in the acquisition of literacy ». Reading Research Quarterly, n²1, p.360-407.

STANOVICH K. E. \& CUNNINGHAM A. E. (1993). «Where does knowledge come from? Specific associations between print exposure and information acquisition ». Journal of Educational Psychology, n85, p. 211-229.
STUEBING K. K., BARTH A. E., MOLFESE P. J., WEISS B. \& FLETCHER J. M. (2009). «IQ is not strongly related to response to reading instruction: A meta-analytic interpretation». Exceptional Children, ${ }^{\circ}$ 76(1), p. 31-51.

THEUREL A. GENTAZ E. \& SPRENGER-CHAROLLES L. (2017). Manuel de passation et de cotation des épreuves utilisées dans les études de Gentaz et al. (2013 et 2015). Genève : FAPSE-UNIGE. En ligne : <https://www.unige.ch/fapse/ sensori-moteur/files/8614/9309/9601/LivretEvaluerLectureGentazTheurelSprenger-Charolles2017.pdf> (consulté le 2 février 2018).

TILSTRA J., MCMASTER K., VAN DEN BROEK P., KENDEOU P. \& RAPP D. (2009). «Simple but complex: Components of the simple view of reading across grade levels ». Journal of research in reading, $n^{\circ} 32(4)$, p.383-401.

TUNMER W. E. \& CHAPMAN J. W. (2012). «The simple view of reading redux: Vocabulary knowledge and the independent components hypothesis». Journal of Learning Disabilities, $\mathrm{n}^{\circ}$ 45(5), p.453-466.

VAN DEN BROEK P., KENDEOU P., LOUSBERG S. \& VISSER G. (2011). «Preparing for reading comprehension: Fostering text comprehension skills in preschool and early elementary school children ». International Electronic Journal of Elementary Education, $\mathrm{n}^{\circ} 4(1)$, p. 259-268.

VELLUTINO F. R., TUNMER W.E., JACCARD J. J. \& CHEN R. (2007). «Components of Reading Ability: Multivariate Evidence for a Convergent Skills Model of Reading Development ». Scientific Studies of Reading, ${ }^{\circ} 11(1)$, p. 3-32

WAGNER R. K., HERRERA S. K., SPENCER M. \& QUINN J. M. (2014). «Reconsidering the Simple View of Reading in an Intriguing Case of Equivalent Models (Commentary on Tunmer and Chapman 2012) ». Journal of learning disabilities, vol.48, n² 2 . En ligne : <http://journals.sagepub.com/ doi/10.1177/0022219414544544> (consulté le 2 février 2018). 